\title{
Electronic correlations in the iron pnictides
}

\author{
M. M. Qazilbash ${ }^{1 \star}$, J. J. Hamlin ${ }^{1,2}$, R. E. Baumbach ${ }^{1,2}$, Lijun Zhang ${ }^{3}$, D. J. Singh ${ }^{3}$, M. B. Maple ${ }^{1,2}$ \\ and D. N. Basov ${ }^{1}$
}

In correlated metals derived from Mott insulators, the motion of an electron is impeded by Coulomb repulsion due to other electrons. This phenomenon causes a substantial reduction in the electron's kinetic energy, leading to remarkable experimental manifestations in optical spectroscopy'. The hightransition-temperature $\left(T_{c}\right)$ superconducting cuprates are perhaps the most studied examples of such correlated metals. The occurrence of high- $T_{c}$ superconductivity in the iron pnictides ${ }^{2-4}$ puts a spotlight on the relevance of correlation effects in these materials ${ }^{5}$. Here, we present an infrared and optical study on single crystals of the iron pnictide superconductor LaFePO. We find clear evidence of electronic correlations in metallic LaFePO with the kinetic energy of the electrons reduced to half of that predicted by band theory of nearly free electrons. We deduce that electronic many-body effects are important in the iron pnictides despite the absence of a Mott transition.

The recent discovery of superconductivity in the iron pnictides promises to be an important milestone in condensed-matter physics $^{2,3}$. Here is a new class of materials with a layered structure and relatively high superconducting $T_{\mathrm{c}}$ values ${ }^{3,4}$ rivalling the doped cuprates. Electronic conduction is believed to occur in the ironpnictogen layers ${ }^{6}$, similar to the cuprates where the charge carriers are delocalized in the copper-oxygen planes. Two decades of research on the cuprates have established that a proper account of the exotic normal-state properties is a prerequisite for the understanding of the superconducting instability ${ }^{7}$.

The correlated metallic state of the superconducting cuprates is derived through chemical doping of the parent compounds, which are strongly correlated (Mott) insulators. However, the parent compounds of the iron pnictides are metallic, albeit highly dissipative, bad metals ${ }^{5}$. Magnetic ordering in the parent iron arsenides at low temperatures leads to partial gapping of the Fermi surface but does not initiate an insulating state. Moreover, there is no evidence of long-range magnetic ordering in the 1111-iron phosphide LaFePO (ref. 8). Recent theoretical work on the normal state of the iron pnictides suggests that despite the apparent itinerant behaviour, Mott physics is relevant to charge dynamics and magnetic properties, ${ }^{5,9,10}$. Thus motivated, we investigated the normal state of the 1111-iron phosphide superconductor LaFePO with infrared and optical spectroscopy, focusing on charge dynamics in the conducting planes.

An optical experiment measures the dynamical response of the electron subjected to an external electromagnetic field and facilitates monitoring of many-body effects experienced by the electron in a material. These many-body effects include the interaction of the electron with other electrons, phonons as well as ordered or fluctuating spins. Figure la shows the real part of the $a b$-plane optical conductivity $\sigma_{1}(\omega)$ of LaFePO over a broad frequency range. Sample growth and characterization procedures, and the experimental details for obtaining the optical conductivity are provided in the Methods section and Supplementary Information. The low-frequency infrared response is dominated by the narrow Drude feature signifying the presence of itinerant charge carriers. Two distinct interband transitions in the form of hump-like structures are seen at higher frequencies. The temperature dependence of the Drude feature is shown in Fig. 1b. The Drude peak at low frequencies becomes sharper, and the conductivity at the lowest measured frequencies increases with decreasing temperature, which is typical metallic behaviour.

To gain insight into the effect of many-body interactions on the itinerant behaviour of the charge carriers, we carried out the extended Drude analysis ${ }^{11}$. Through the extended Drude analysis, we extract the electronic scattering rate $1 / \tau(\omega)$ and the mass enhancement factor $m^{*}(\omega) / m_{\mathrm{b}}$ (see Supplementary Information). The temperature dependence of $1 / \tau(\omega)$ and $m^{*}(\omega \rightarrow 0) / m_{\mathrm{b}}$ is respectively plotted in Fig. 2 and the inset. It can be seen that the scattering rate increases continuously and monotonically with frequency. Electron-phonon scattering theory predicts a constant, frequency-independent scattering rate above the cutoff of the phonon spectrum ${ }^{11,12}$. Measurements and calculations of the phonon spectral density for LaFePO are not yet available. However, the cutoff is not likely to exceed $550 \mathrm{~cm}^{-1}$ inferred from calculations on the 1111-iron arsenide LaFeAsO (refs 13, 14). Therefore, the absence of saturation of $1 / \tau(\omega)$ at higher frequencies in LaFePO suggests that in addition to electron-phonon scattering, there are other scattering channel(s) arising from electronic and spin correlations. It is interesting to note that the scattering rate has a linear frequency dependence between 1,100 and $2,000 \mathrm{~cm}^{-1}$ and it deviates from linearity below $1,100 \mathrm{~cm}^{-1}$. This frequency dependence almost mimics the temperature dependence of the resistivity, which has a linear temperature dependence at higher temperatures and a quadratic temperature dependence at low temperatures ${ }^{15}$.

At $T=10 \mathrm{~K}$, the frequency-dependent scattering rate lies below the line $\omega=1 / \tau(\omega)$ at low frequencies, signifying the presence of coherent quasiparticles. This is consistent with the observation of de Haas-van Alphen oscillations in LaFePO (ref. 16). Note, however, that $1 / \tau(\omega)$ is of the order of the excitation energy (frequency) at high energies (frequencies) even at low temperatures. This is significant because quasiparticles may be well defined only near the Fermi energy, whereas at higher energies we are dealing with a strongly dissipative system. At higher temperatures, $1 / \tau(\omega)$ approaches the line $\omega=1 / \tau(\omega)$, that is, the incoherent regime. In doped iron arsenides, the scattering rate exceeds the excitation frequency ${ }^{17}$, implying that transport in the iron arsenides is more incoherent than in its phosphorous-based counterparts. We note that the mass enhancement in the zero-frequency

\footnotetext{
${ }^{1}$ Department of Physics, University of California, San Diego, La Jolla, California 92093, USA, ${ }^{2}$ Institute for Pure and Applied Physical Sciences, University of California, San Diego, La Jolla, California 92093, USA, ${ }^{3}$ Materials Science and Technology Division, Oak Ridge National Laboratory, Oak Ridge, Tennessee 37831, USA. *e-mail: mumtaz@physics.ucsd.edu.
} 

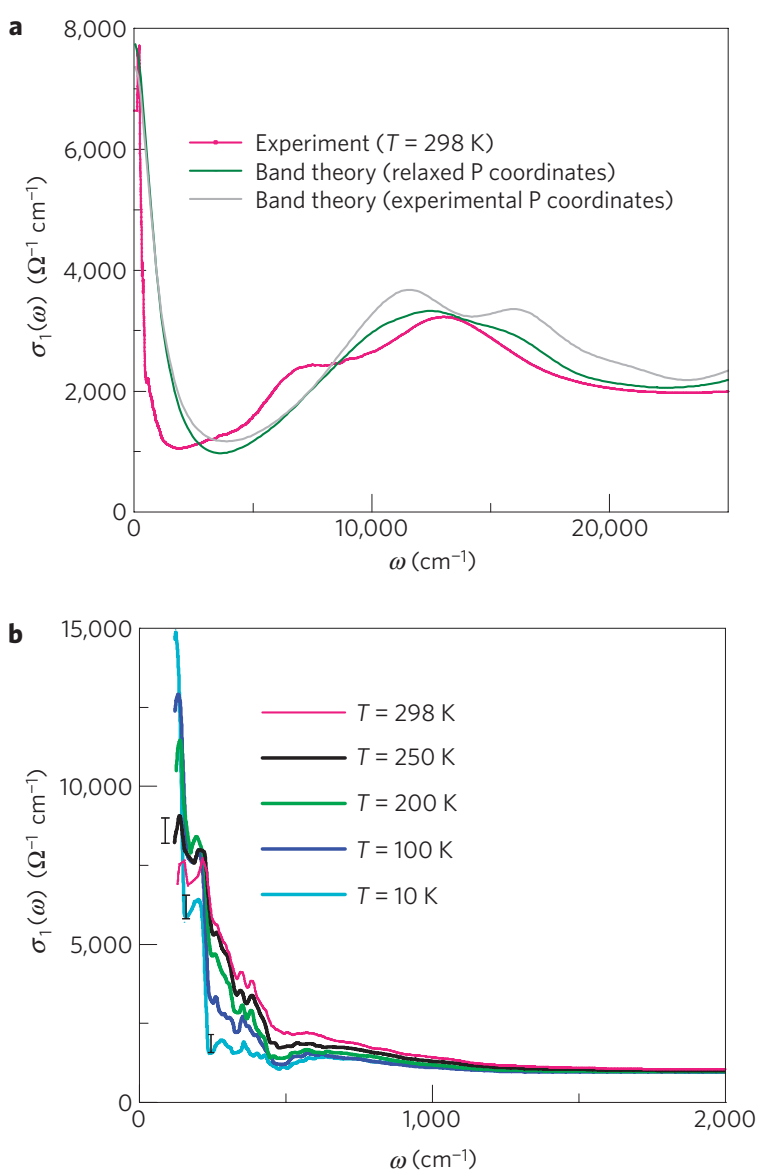

Figure 1 | $\boldsymbol{a b}$-plane optical conductivity of LaFePO. a, Real part of the $a b$-plane optical conductivity $\sigma_{1}(\omega)$ of LaFePO as a function of frequency for $T=298 \mathrm{~K}$. Also shown are $\sigma_{1}(\omega)$ plots calculated within band theory for two choices of the phosphorous $(P)$ coordinate. $\mathbf{b}$, Systematic temperature dependence of $\sigma_{1}(\omega)$. The uncertainty in $\sigma_{1}(\omega)$ in $\mathbf{b}$ is less than or equal to the thickness of the lines for $\omega>350 \mathrm{~cm}^{-1}$. However, the uncertainty in $\sigma_{1}(\omega)$ increases at lower frequencies as indicated by the error bars.

limit due to low-energy many-body interactions is at most 1.5 (Fig. 2, inset). This value of the mass enhancement is obtained using the experimentally determined plasma frequency, which is already renormalized by high-energy electronic correlations (see subsequent discussion and Supplementary Information). Therefore, the mass enhancement obtained from the extended Drude analysis is a result of low-energy many-body interactions. On the other hand, we find that high-energy electronic correlations lead to an effective mass renormalization in LaFePO by a factor of 2 , which is equivalent to a kinetic energy reduction by a factor of 2 compared with the band theory value, which we discuss next.

With the itinerant nature of LaFePO firmly identified by our data in Figs 1 and 2, we now proceed to the central part of our analysis pertaining to the electronic kinetic energy. The optical conductivity data provide a measure of the kinetic energy $K$ of the electrons ${ }^{1}$. The kinetic energy can also be readily evaluated using band-structure calculations. As a rule, experimental results for itinerant electron systems are in good agreement with the band-structure calculations leading to $K_{\exp } / K_{\text {band }}$ close to unity in simple metals (see Fig. 3 ). However, interesting electronic effects due to strong interactions involving charge, spin and orbital degrees of freedom occurring in many intermetallic compounds with $d$ - (and $f$-) electrons yield dynamical effects beyond ordinary band-structure results. All of these effects compete with itinerancy of electrons, leading to the suppression of the $K_{\exp } / K_{\text {band }}$ value from unity. The most

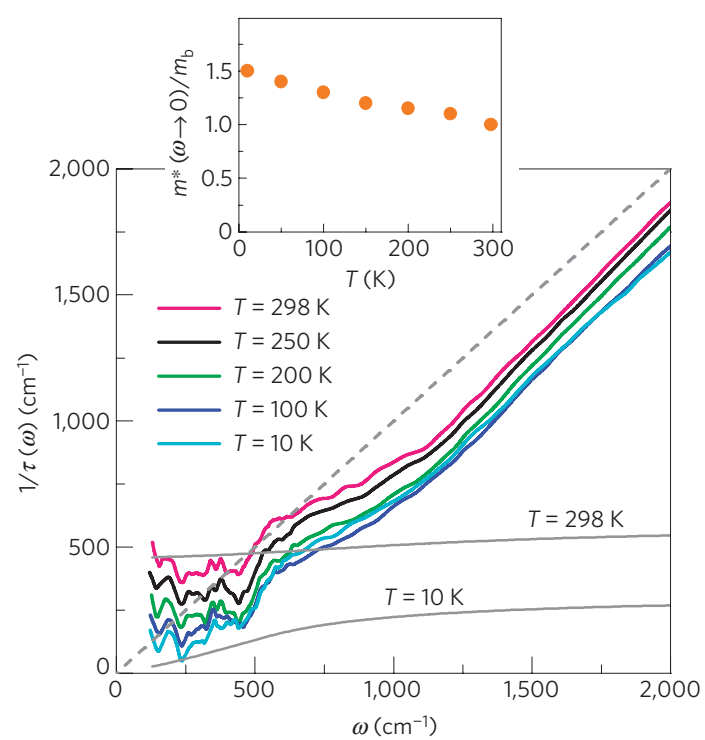

Figure 2 | The frequency-dependent scattering rate and the mass enhancement factor in the low-frequency limit. The frequency dependence of the scattering rate $1 / \tau(\omega)$ for representative temperatures in the normal state of LaFePO. The dashed line represents the equation $\omega=1 / \tau(\omega)$. The solid grey lines are the expected electron-phonon scattering rates at $T=298$ and $10 \mathrm{~K}$ calculated using a model phonon spectral density for LaFePO (see Supplementary Information). The inset shows the temperature dependence of the mass enhancement factor in the low frequency limit $m^{*}(\omega \rightarrow 0) / m_{b}$.

notorious example is a Mott insulator where $K_{\exp }=0$ due to Coulomb repulsion, whereas band-structure results still predict metallic response with finite $K_{\text {band }}$. A trend revealed by metals in the vicinity of Mott insulators is that $K_{\exp } / K_{\text {band }}$ increases from zero to finite values as Coulomb correlations are suppressed, resulting in a Mott insulator-to-metal transition with doping or temperature (Fig. 3). Although $K_{\text {exp }} / K_{\text {band }}$ in correlated metals derived from Mott insulators is finite, it is significantly less than unity. Therefore, optical experiments in tandem with band-structure results offer reliable means to probe electronic correlations in materials.

We have calculated $\sigma_{1}(\omega)$ from band theory within the generalized gradient approximation using both experimental and relaxed phosphorous coordinates (see Fig. 1a and Supplementary Information). The calculated $\sigma_{1}(\omega)$ shows a Drude feature at low frequencies and higher-frequency interband transitions, in reasonable agreement with the experimental data. The band-theory calculations show that $\mathrm{LaFePO}$ is a metal in agreement with experiment. There are, however, some differences between the calculated and measured $\sigma_{1}(\omega)$ that will be elaborated in subsequent paragraphs. We note here that generalized gradient approximation calculations were carried out with two choices of the atomic coordinates for phosphorous. The two choices do not significantly affect the Drude part but do affect the peak positions of the interband transitions (Fig. 1a).

We now wish to draw attention to the key parameter associated with the optical conductivity - the area under the Drude part of $\sigma_{1}(\omega)$, which is proportional to the electron's kinetic energy (or the plasma frequency squared). It can be immediately seen in Fig. 1a that the area under the Drude part of the measured $\sigma_{1}(\omega)$ is significantly less than that calculated within band theory. To put this on a quantitative footing, we define the experimental kinetic energy as ${ }^{18}$ :

$$
K_{\exp }\left(\omega_{\mathrm{c}}\right)=\frac{\hbar c_{0}}{e^{2}} \int_{0}^{\omega_{\mathrm{c}}} \frac{2 \hbar}{\pi} \sigma_{1}(\omega) \mathrm{d} \omega
$$




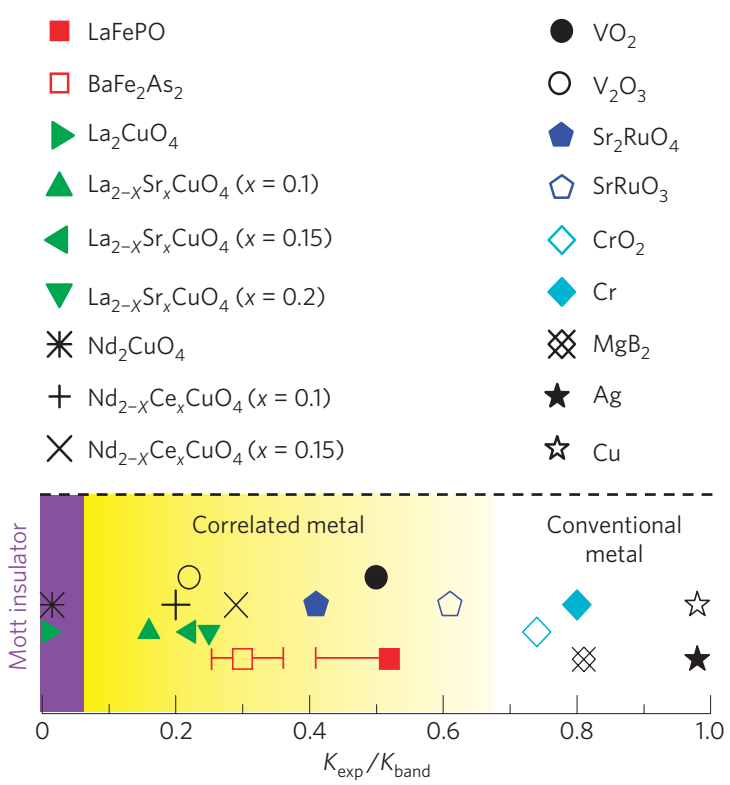

Figure 3 | Ratio of the experimental kinetic energy and the kinetic energy from band theory $K_{\text {exp }} / K_{\text {band }}$ for the iron pnictides and various other materials. The data points are offset in the vertical direction for clarity. The value of the LaFePO data point and the associated error bar are discussed in the text, the Methods section and Supplementary Information. The values of $K_{\text {exp }}$ and $K_{\text {band }}$ for other materials are obtained from the following references: paramagnetic $\mathrm{BaFe}_{2} \mathrm{As}_{2}$ (refs 24-26), $\mathrm{La}_{2-x} \mathrm{Sr}_{x} \mathrm{CuO}_{4}$ (ref. 20), $\mathrm{Nd}_{2-x} \mathrm{Ce}_{x} \mathrm{CuO}_{4}$ (ref. 20), $\mathrm{VO}_{2}$ rutile metal (ref. 12), $\mathrm{V}_{2} \mathrm{O}_{3}$ paramagnetic metal (refs 27, 28), $\mathrm{Sr}_{2} \mathrm{RuO}_{4}$ (refs 29, 30), paramagnetic

$\mathrm{SrRuO}_{3}$ (refs 31, 32), half-metallic $\mathrm{CrO}_{2}$ (refs 33-35), paramagnetic $\mathrm{Cr}$ (ref. 36), $\mathrm{MgB}_{2}$ (same data point for $\mathrm{a}$ and $\mathrm{c}$ axes) (ref. 37), $\mathrm{Ag}$ and $\mathrm{Cu}$ (ref. 38). The error bar on the $\mathrm{BaFe}_{2} \mathrm{As}_{2}$ data point is based on the scatter in the theory and experimental values of the plasma frequency

(refs 24-26). Error bars (not shown) for other metals are estimated to be at least the width of the symbols and at most \pm 0.05 .

In equation (1), $c_{0}$ is the distance between the FeP planes. On integrating the Drude part of the experimental $\sigma_{1}(\omega)$ up to a cutoff frequency $\omega_{\mathrm{c}}=3,000 \mathrm{~cm}^{-1}$, we obtain a kinetic energy $K_{\exp }=0.15 \mathrm{eV}$. The theoretical value of the kinetic energy is obtained directly from band-theory calculations of the plasma frequency, which give $K_{\mathrm{band}}=0.29 \mathrm{eV}$ (see Supplementary Information for details). $K_{\text {exp }}$ is nearly $50 \%$ of $K_{\text {band }}$ and this reduction is due to correlation effects not accounted for in band theory. We note that this discrepancy between $K_{\exp }$ and $K_{\text {band }}$ is robust with respect to the uncertainties in the experimental and theoretical values of the kinetic energy, which are discussed in the Methods section and Supplementary Information. Furthermore, our findings are in agreement with a recent photoemission experiment in which the measured bandwidth is nearly half of that calculated within band theory ${ }^{19}$.

Among the various superconducting iron pnictide families, $\mathrm{LaFePO}$ has one of the highest normal-state conductivities. Despite the high conductivity, the reduction of the kinetic energy is significant in what is probably the least correlated material among the iron pnictides. Therefore, the ground-state wavefunction of $\mathrm{LaFePO}$ is not the same as calculated by band theory, and dynamical correlation effects omitted by band theory have to be taken into account for a realistic theoretical description. This view is further supported by the differences between the measured optical interband transitions and those derived from the band structure (see Fig. 1a). In the experimental data, there are two distinct peaks in $\sigma_{1}(\omega)$ centred at 7,200 and $13,000 \mathrm{~cm}^{-1}$, most likely arising from optical transitions between the Fe- $3 d$ bands. We note that the band-theory calculations predict two peaks between 10,000 and $17,000 \mathrm{~cm}^{-1}$. The discrepancy between the experimental data and band-structure calculations is probably due to modification of the actual band structure arising from electronic correlations.

The reduction of the kinetic energy compared with band theory in LaFePO, its proximity to the incoherent metal regime and the absence of a Mott insulator in its phase diagram inspired us to compare it with other exotic conductors that have been studied in the past. Figure 3 is a compilation showing the location of various exotic conductors as well as a few conventional metals ( $\mathrm{Ag}$ and $\mathrm{Cu}$ ) based on the ratio of the experimental kinetic energy to that calculated within band theory. The exotic conductors that have been selected for comparison share at least one of the following features in common with the iron pnictides: high- $T_{\mathrm{c}}$ superconductivity, itinerant magnetism and/or electronic correlations. Within the ambit of Fig. 3, the correlated metal regime is characterized by a substantially reduced empirical kinetic energy of charge carriers compared with theory. Note that electron-phonon coupling does not result in a significant reduction of the electron's kinetic energy (unless it is extremely strong as to lead to polaron formation $)^{1}$. Electron-phonon coupling in $\mathrm{MgB}_{2}$ superconductor, for example, results in $K_{\text {exp }} / K_{\text {band }}$ not much less than unity in the metallic state (Fig. 3).

We now look at the consequences of magnetic interactions on the kinetic energy. In paramagnetic $\mathrm{Cr}$ and half-metallic $\mathrm{CrO}_{2}$, two classic systems with itinerant magnetism, spin correlations lead to $20-25 \%$ reduction in $K_{\exp }$ relative to $K_{\text {band }}$ (Fig. 3). Moreover, in the cuprates where the magnetism has local moment character, opposite to the itinerant case discussed above, magnetic interactions also lead to a relatively small reduction in the electron's kinetic energy compared with that predicted by band theory ${ }^{20}$. Even where it is argued that the role of magnetic interactions in the cuprates is important, it is seen that $80-90 \%$ of the experimental reduction in kinetic energy compared with band theory is accounted for by the intra-atomic Coulomb repulsion and $10-20 \%$ may be attributed to antiferromagnetic correlation $s^{20}$. It is worth emphasizing that $K_{\exp }$ is reduced substantially compared with $K_{\text {band }}$ in the cuprates, oxides of vanadium and the ruthenate family of metals as shown in Fig. 3these materials have Mott insulators in their phase diagrams and dynamical Coulomb correlations dominate transport behaviour in the metallic states. Thus, intra-atomic Coulomb repulsion is one established mechanism that leads to a substantial reduction in $K_{\exp }$ compared with $K_{\text {band }}$.

We note that the parent compounds of the superconducting iron arsenides exhibit long-range magnetic ordering at low temperature ${ }^{21}$, but they remain metallic and are not insulating in the ordered state. Furthermore, $\mathrm{LaFePO}$ shows no signs of long-range magnetic ordering ${ }^{8}$ or insulating behaviour ${ }^{15}$. However, spin correlations probably exist in $\mathrm{LaFePO}$ and the paramagnetic phases of the iron arsenides ${ }^{13}$. The data in Fig. 3 show that both in $\mathrm{LaFePO}$ and paramagnetic $\mathrm{BaFe}_{2} \mathrm{As}_{2}, K_{\text {exp }} / K_{\text {band }}$ is less than that in other typical itinerant magnets and is substantially less than unity. Notice that $K_{\text {exp }} / K_{\text {band }}$ is further reduced for paramagnetic $\mathrm{BaFe}_{2} \mathrm{As}_{2}$ compared with LaFePO, which means that the arsenides are even more correlated than the phosphides ${ }^{5}$. These observations may not be explained by spin correlations alone, in which case they signify the relevance of dynamical Coulomb correlations to the physics of the iron pnictides. This is remarkable because so far there is no evidence of a Mott transition in their phase diagrams. The iron pnictides are properly classified as being in the moderate correlation regime with the on-site Coulomb repulsion of the order of the bandwidth. Our results demonstrate that transport in the iron pnictides lies between the band-like itinerant and the Mott-like local magnetic moment extremes. This implies the presence of both coherent and incoherent excitations in the iron pnictides ${ }^{22}$. 


\section{Methods}

The growth and characterization procedures for LaFePO single crystals are given in ref. 15. Resistivity and magnetization measurements reveal a superconducting $T_{\mathrm{c}}$ of $\approx 6 \mathrm{~K}$, with complete zero-field-cooled diamagnetic shielding in the superconducting state. The crystals are platelets, typically $0.5 \mathrm{~mm} \times 0.5 \mathrm{~mm} \times 0.05 \mathrm{~mm}$ in size.

The $a b$-plane reflectance was measured in the near-normal incidence geometry in a Bruker v66 Infrared Fourier-transform Spectrometer at frequencies between 100 and $24,000 \mathrm{~cm}^{-1}$. The reflectance measurements were carried out at the following temperatures: $298,250,200,150,100,50$ and $10 \mathrm{~K}$. The temperature dependence of the $a b$-plane reflectance of LaFePO crystals is shown in Supplementary Fig. S1. We obtained the optical constants through fitting the reflectance data with Drude and Lorentzian oscillators, and Kramers-Kronig constrained variational dielectric functions as described in ref. 23. In addition, variable-angle spectroscopic ellipsometry was carried out in the frequency range $5500-25,000 \mathrm{~cm}^{-1}$, which improves the accuracy of the extracted optical constants in this frequency range.

Details regarding the extended Drude analysis are given in Supplementary Information. We note here that $K_{\exp } / K_{\text {band }}=0.52$ for LaFePO plotted in Fig. 3 is an upper bound, and other methods for obtaining $K_{\exp } / K_{\text {band }}$ give a lower bound of 0.4. Further details about calculations of $K_{\text {exp }}$ and $K_{\text {band }}$ for $\mathrm{LaFePO}$ are given in Supplementary Information. Also included in Supplementary Information are calculations of the scattering rates due to electron-phonon coupling, and some notes on Fig. 3.

\section{Received 3 February 2009; accepted 23 June 2009;} published online 26 July 2009

\section{References}

1. Millis, A. J. in Strong Interactions in Low Dimensions (eds Baeriswyl, D. \& Degiorgi, L.) (Kluwer-Academic, 2004).

2. Kamihara, Y. et al. Iron-based layered superconductor: LaOFeP. J. Am. Chem. Soc. 128, 10012-10013 (2006).

3. Kamihara, Y., Watanabe, T., Hirano, M. \& Hosono, H. Iron-based layered superconductor $\mathrm{LaO}_{1-x} \mathrm{~F}_{x} \mathrm{FeAs}(x=0.05-0.12)$ with $T_{\mathrm{c}}=26 \mathrm{~K}$. J. Am. Chem. Soc. 130, 3296-3297 (2008).

4. Ren, Z.-A. et al. Superconductivity at $55 \mathrm{~K}$ in iron-based F-doped layered quaternary compound $\mathrm{SmO}_{1-x} \mathrm{~F}_{x}$ FeAs. Chin. Phys. Lett. 25, 2215-2216 (2008)

5. Si, Q. \& Abrahams, E. Strong correlations and magnetic frustration in the high $T_{\mathrm{c}}$ iron pnictides. Phys. Rev. Lett. 101, 076401 (2008).

6. Kamihara, Y. et al. Electromagnetic properties and electronic structure of the iron-based layered superconductor LaFePO. Phys. Rev. B 77, 214515 (2008).

7. Bonn, D. A. Are high-temperature superconductors exotic? Nature Phys. 2, 159-168 (2006).

8. Carlo, J. P. et al. Static magnetic order and superfluid density of RFeAs(O, F) $(\mathrm{R}=\mathrm{La}, \mathrm{Nd}, \mathrm{Ce})$ and $\mathrm{LaFePO}$ studied by muon spin relaxation: Unusual similarities with the behavior of cuprate superconductors. Phys. Rev. Lett. 102, 087001 (2009).

9. Haule, K., Shim, J. H. \& Kotliar, G. Correlated electronic structure of $\mathrm{LaO}_{1-x} \mathrm{~F}_{x}$ FeAs. Phys. Rev. Lett. 100, 226402 (2008).

10. Laad, M. S., Craco, L., Leoni, S. \& Rosner, H. Electrodynamic response of incoherent metals: Normal phase of iron pnictides. Phys. Rev. B 79, 024515 (2009)

11. Basov, D. N. \& Timusk, T. Electrodynamics of high- $T_{\mathrm{c}}$ superconductors. Rev. Mod. Phys. 77, 721-779 (2005).

12. Qazilbash, M. M. et al. Correlated metallic state of vanadium dioxide. Phys. Rev. B 74, 205118 (2006).

13. Singh, D. J. \& Du, M.-H. Density functional study of $\mathrm{LaFeAsO}_{1-x} \mathrm{~F}_{x}$ : A low carrier density superconductor near itinerant magnetism. Phys. Rev. Lett. 100, 237003 (2008)

14. Boeri, L., Dolgov, O. V. \& Golubov, A. A. Is $\mathrm{LaFeAsO}_{1-x} \mathrm{~F}_{x}$ an electron-phonon superconductor? Phys. Rev. Lett. 101, 026403 (2008).

15. Hamlin, J. J., Baumbach, R. E., Zocco, D. A., Sayles, T. A. \& Maple, M. B. Superconductivity in single crystals of LaFePO. J. Phys. Condens. Matter 20, 365220 (2008).

16. Coldea, A. I. et al. Fermi surface of superconducting LaFePO determined from quantum oscillations. Phys. Rev. Lett. 101, 216402 (2008).

17. Yang, J. et al. Optical spectroscopy of superconducting $\mathrm{Ba}_{0.55} \mathrm{~K}_{0.45} \mathrm{Fe}_{2} \mathrm{As}_{2}$ : Evidence for strong coupling to low-energy bosons. Phys. Rev. Lett. 102, 187003 (2009)
18. Millis, A. J., Zimmers, A., Lobo, R. P. S. M., Bontemps, N. \& Homes, C. C. Mott physics and the optical conductivity of electron-doped cuprates. Phys. Rev. B 72, 224517 (2005).

19. Lu, D. H. et al. Electronic structure of the iron-based superconductor LaOFeP. Nature 455, 81-84 (2008).

20. Comanac, A., De Medici, L., Capone, M. \& Millis, A. J. Optical conductivity and the correlation strength of high-temperature copper-oxide superconductors. Nature Phys. 4, 287-290 (2008).

21. de la Cruz, C. et al. Magnetic order close to superconductivity in the iron-based layered $\mathrm{LaO}_{1-x} \mathrm{~F}_{x} \mathrm{FeAs}$ systems. Nature 453, 899-902 (2008).

22. Si, Q., Abrahams, E., Dai, J. \& Zhu, J.-X. Correlation effects in the iron pnictides. N. J. Phys. 11, 045001 (2009).

23. Kuzmenko, A. B. Kramers-Kronig constrained variational analysis of optical spectra. Rev. Sci. Instrum. 76, 083108 (2005).

24. $\mathrm{Hu}, \mathrm{W}$. Z. et al. Origin of the spin density wave instability in $\mathrm{AFe}_{2} \mathrm{As}_{2}$ $(\mathrm{A}=\mathrm{Ba}, \mathrm{Sr})$ as revealed by optical spectroscopy. Phys. Rev. Lett. 101, 257005 (2008).

25. Ma, F., Lu, Z.-Y. \& Xiang, T. Electronic structures of ternary iron arsenides $\mathrm{AFe}_{2} \mathrm{As}_{2}(\mathrm{~A}=\mathrm{Ba}, \mathrm{Ca}$, or Sr). Preprint at <http://arxiv.org/abs/0806.3526> (2008).

26. Drechsler, S.-L. et al. New insight into the physics of iron pnictides from optical and penetration depth data. Preprint at <http://arxiv.org/abs/0904.0827> (2009).

27. Thomas, G. A. et al. Optical properties of a correlated electron system. J. Low Temp. Phys. 95, 33-38 (1994).

28. Rozenberg, M. J., Kotliar, G. \& Kajueter, H. Transfer of spectral weight in spectroscopies of correlated electron systems. Phys. Rev. B 54, 8452-8468 (1996).

29. Katsufuji, T., Kasai, M. \& Tokura, Y. In-plane and out-of-plane optical spectra of $\mathrm{Sr}_{2} \mathrm{RuO}_{4}$. Phys. Rev. Lett. 76, 126-129 (1996).

30. Singh, D. J. Relationship of $\mathrm{Sr}_{2} \mathrm{RuO}_{4}$ to the layered superconducting cuprates. Phys. Rev. B 52, 1358-1361 (1995).

31. Ahn, J. S. et al. Spectral evolution in $(\mathrm{Ca}, \mathrm{Sr}) \mathrm{RuO}_{3}$ near the Mott-Hubbard transition. Phys. Rev. Lett. 82, 5321-5324 (1999).

32. Mazin, I. I. \& Singh, D. J. Electronic structure and magnetism in Ru-based perovskites. Phys. Rev. B 56, 2556-2571 (1997).

33. Singley, E. J., Weber, C. P., Basov, D. N., Barry, A. \& Coey, J. M. D Charge dynamics in the half-metallic ferromagnet $\mathrm{CrO}_{2}$. Phys. Rev. B 60, 4126-4130 (1999).

34. Lewis, S. P., Allen, P. B. \& Sasaki, T. Band structure and transport properties of $\mathrm{CrO}_{2}$. Phys. Rev. B 55, 10253-10260 (1997).

35. Mazin, I. I., Singh, D. J. \& Ambrosch-Draxl, C. Transport, optical, and electronic properties of the half-metal $\mathrm{CrO}_{2}$. Phys. Rev. B 59, 411-418 (1999).

36. Romaniello, P., de Boeij, P. L., Carbone, F. \& van der Marel, D. Optical properties of bcc transition metals in the range $0-40 \mathrm{eV}$. Phys. Rev. B 73, 075115 (2006).

37. Guritanu, V. et al. Anisotropic optical conductivity and two colors of $\mathrm{MgB}_{2}$. Phys. Rev. B 73, 104509 (2006).

38. Romaniello, P. \& de Boeij, P. L. Time-dependent current-density-functional theory for the metallic response of solids. Phys. Rev. B 71, 155108 (2005).

\section{Acknowledgements}

The authors are grateful to E. Abrahams, A. V. Boris, A. V. Chubukov, A. J. Millis, O. Shpyrko, Q. Si and C. Wu for discussions. M.M.Q. thanks A. Kuzmenko for assistance with the software for infrared data analysis. D.N.B. acknowledges support from the National Science Foundation (grant NSF DMR 0705171) and M.B.M. acknowledges support from the Department of Energy (grant DE-FG02-04ER46105).

\section{Author contributions}

M.M.Q. carried out the infrared and optical experiments and the data analysis. M.M.Q. wrote the paper with input from all of the other co-authors, especially from D.N.B. J.J.H. and R.E.B. were responsible for the growth and characterization of the LaFePO single crystals. L.Z. and D.J.S. carried out band-structure calculations for LaFePO and calculated its plasma frequency and optical conductivity. D.N.B. and M.B.M. were responsible for the planning and management of the project.

\section{Additional information}

Supplementary information accompanies this paper on www.nature.com/naturephysics. Reprints and permissions information is available online at http://npg.nature.com/ reprintsandpermissions. Correspondence and requests for materials should be addressed to M.M.Q. 